\title{
Gravitational particle creation for dark matter and reheating
}

\author{
Soichiro Hashiba ${ }^{1,2, *}$ and Jun'ichi Yokoyama ${ }^{1,2,3, \dagger}$ \\ ${ }^{1}$ Department of Physics, Graduate School of Science, The University of Tokyo, Tokyo 113-0033, Japan \\ ${ }^{2}$ Research Center for the Early Universe (RESCEU), Graduate School of Science, \\ The University of Tokyo, Tokyo 113-0033, Japan \\ ${ }^{3}$ Kavli Institute for the Physics and Mathematics of the Universe (Kavli IPMU), WPI, UTIAS, \\ The University of Tokyo, 5-1-5 Kashiwanoha, Kashiwa 277-8583, Japan
}

(Received 29 December 2018; published 20 February 2019)

\begin{abstract}
The purely gravitational dark matter (PGDM) which interacts with the standard model particles only by gravitational interaction is a topic of recent discussion. Due to its feeble interaction, PGDM may be produced mainly by the gravitational particle creation, which plays an important role in the reheating after kinetically driven inflation and in some potential-driven inflation without a subsequent inflaton oscillating phase. Therefore, we consider the possibility of the gravitational reheating model which can simultaneously explain the present PGDM density. We consider a model where two massive scalar fields are incorporated into the standard model in addition to the inflation sector. We show that the gravitational particle creation prevails over the thermal creation - the freeze-in process — and it can actually explain the reheating and the present abundance of dark matter if one of the scalar particles is as heavy as the Hubble parameter during inflation $\sim 10^{13} \mathrm{GeV}$ and finally decays into radiation via sufficiently weak coupling and the other is a stable PGDM with mass of the order of $10^{3} \mathrm{GeV}$.
\end{abstract}

DOI: 10.1103/PhysRevD.99.043008

\section{INTRODUCTION}

Although the $\Lambda$ CDM model explains the evolution of the Universe quite well, the identity of cold dark matter (CDM) and its production mechanism are still missing pieces in modern cosmology. According to the results of the cosmic microwave background (CMB) observation [1], the dark matter occupies about $27 \%$ of the total energy density of the Universe. It must not interact with the standard model (SM) particles through electromagnetic interactions since the dark matter is "invisible." Therefore, it is natural to consider the weakest interacting dark matter which interacts with the SM particles only gravitationally [2-5]. Such dark matter is called the Planckian interacting dark matter (PIDM) [6] and also the pure(ly) gravitational dark matter (PGDM) [7,8]. We use the latter terminology hereafter. PGDM is difficult to create due to its extremely weak interaction.

The reheating process after inflation remains a problem in cosmology. The inflationary universe model (see, e.g., Ref. [9] for a review of cosmic inflation.) has amazingly

\footnotetext{
sou16.hashiba@resceu.s.u-tokyo.ac.jp

yokoyama@resceu.s.u-tokyo.ac.jp
}

Published by the American Physical Society under the terms of the Creative Commons Attribution 4.0 International license. Further distribution of this work must maintain attribution to the author(s) and the published article's title, journal citation, and DOI. Funded by SCOAP. succeeded in explaining the very early universe and the later structure formation by providing an appropriate initial condition of primodial fluctuations. However, how to realize the reheating process - the particle creation after inflation which turns the inflaton-dominated universe into the radiation-dominated-is not yet completely understood. Inflationary models are roughly classified into two types: those driven by a potential energy of the inflaton and those driven by its kinetic energy. In most potentialdriven models [10-13], the reheating can be realized by the inflaton field oscillation [14,15]. In kinetically driven models [16,17] and some potential-driven models [18], the inflaton field does not oscillate after inflation, but kination - the epoch when the kinetic energy of a free scalar field dominates the cosmological energy densityfollows inflation and, thus, the reheating model mentioned above is not valid. Therefore, it has been claimed that the reheating process is achieved by the gravitational particle creation in these inflationary models [16-19]. ${ }^{1}$

It is known that any particles that are not conformally invariant are created when the time dependence of the cosmic expansion changes [21,22]. This process is called the gravitational particle creation. Although it has usually been analyzed perturbatively for massive scalar particles

\footnotetext{
${ }^{1}$ The reheating through direct interactions between the inflaton and a matter field preserving shift symmetry has been discussed in Ref. [20].
} 
[8,23-26], in Ref. [27] we have calculated the gravitationally produced energy density nonperturbatively. The gravitational particle creation is suitable for the production of PGDM and also for the reheating after inflation if the inflation field oscillation does not follow (while it is negligible in usual potential-driven inflationary models because it is much less efficient than the decay of coherent inflaton oscillations). Therefore, we examine whether or not the gravitational particle creation can explain the reheating and the production of PGDM at the same time in inflationary models where kination follows inflation, such as in Refs. [16-18].

This paper is organized as follows. Our previous calculation of the gravitational particle creation [27] is briefly reviewed in Sec. II. The present abundance of gravitationally produced PGDM is calculated in Sec. III. PGDM should be produced sufficiently in order to prevent gravitons from disturbing CMB. This issue is discussed in Sec. IV. Since the freeze-in process also produces PGDM, the gravitational particle creation is compared with it in Sec. V. Our results are summarized in Sec. VI. We use the natural units $c=\hbar=1$ and $M_{\mathrm{Pl}}=1.2 \times 10^{19} \mathrm{GeV}$ throughout the paper.

\section{GRAVITATIONAL PARTICLE CREATION AFTER INFLATION}

We consider inflationary models in which the kination stage follows inflation, such as $k$-inflation [16] and kinetically driven G-inflation [17], and adopt the adiabatic vacuum as the basis for counting the number of particles. The concept of the adiabatic vacuum is that the mode function of the vacuum should approach the positive frequency mode in the Minkowski spacetime since, for high-momentum particles, the Universe looks as if it is almost flat and static for the relevant short time and length scales. The equation of motion of a mode function for a scalar field conformally coupled to gravity in a spatially flat Friedmann-Lemaître-Robertson-Walker (FLRW) metric is

$$
\frac{d^{2} \chi_{k}(\eta)}{d \eta^{2}}+\left[k^{2}+a^{2}(\eta) m^{2}\right] \chi_{k}(\eta)=0
$$

where $\eta$ and $a(\eta)$ denote the conformal time and the scale factor, respectively. During de Sitter inflation and kination, the scale factor is asymptotically proportional to $(-\eta)^{-1}$ with $\eta<0$ and $\eta^{1 / 2}$ with $\eta>0$, respectively. Equation (1) can be analytically solved with this scale factor, and the adiabatic vacuum is obtained by imposing the condition

$$
\lim _{k \rightarrow \infty} \chi_{k}(\eta)=\frac{1}{\sqrt{2 k}} e^{-i k \eta}
$$

on the analytic solutions of Eq. (1). The adiabatic vacuum can be written in terms of Hankel functions. We have given an exact form in Ref. [27]. The produced number density is obtained from the Bogoliubov coefficient of the transformation between this adiabatic vacuum and the mode function of the Universe.

Assuming a smooth transition from inflation to kination with time scale $\Delta t$, the energy density of produced particles is given by [27]

$$
\rho=\mathcal{C} e^{-4 m \Delta t} m^{2} H_{\mathrm{inf}}^{2},
$$

where $\mathcal{C} \simeq 2 \times 10^{-4}$, and $m$ and $H_{\text {inf }}$ denote the mass of the created scalar particle and the Hubble parameter during inflation, respectively. As seen in Eq. (3), the mass threshold above which the gravitational particle creation is exponentially suppressed is given by the inverse transition time scale $(\Delta t)^{-1}$ rather than the Hubble parameter during inflation $H_{\text {inf. }}$. Since its power spectrum has a peak around $k \sim m$, the heaviest produced particle soon becomes nonrelativistic by the subsequent expansion of the Universe.

\section{REHEATING AND GRAVITATIONAL PRODUCTION OF DARK MATTER}

We incorporate two massive scalar particles, both of which are conformally coupled to gravity. Only their mass terms violate the conformal symmetry and serve as a source of gravitational particle production. One of them decays into radiation and realizes reheating, and the other is a stable PGDM. We call them $A$ and $X$, respectively. Several papers consider a $\mathrm{U}(1)$ interaction between PGDMs $[28,29]$, but here we do not assume any interaction between $X$ 's other than gravitational interaction. We assume the decay of $A$ and its decay width given as

$$
\Gamma=\alpha m_{A},
$$

where $\alpha$ is a dimensionless constant. For example, $\alpha$ takes $\lambda^{2} / 32 \pi^{2}$ when $A$ decays into a Fermion pair via a Yukawainteraction term $\lambda A \bar{\Psi} \Psi$. Since $H \propto a^{-3}$ during kination, the scale factor at $t=t_{d}$ when $H=\Gamma$ is

$$
a_{d}=\alpha^{-1 / 3}\left(\frac{m_{A}}{H_{\mathrm{inf}}}\right)^{-1 / 3} .
$$

Here and hereafter, we put the scale factor at the end of inflation to unity. Although $A$ constantly decays into radiation until $t_{d}$, the energy density of $A$ is diluted as $a^{-3}$ more slowly than that of radiation as $a^{-4}$ and then it is enough to consider decay of $A$ around $t=t_{d}$ where resultant radiation is least diluted. Here, we assume that $A$ decays during kination. If $A$ does not decay until kination ends, the scale factor at the end of kination is

$$
a_{\mathrm{MD}}=1.0 \times 10^{5} e^{\frac{4}{3} m_{A} \Delta t}\left(\frac{m_{A}}{10^{13} \mathrm{GeV}}\right)^{-2 / 3} .
$$


If $a_{d}<a_{\mathrm{MD}}$, then $A$ actually decays during kination. According to Eqs. (5) and (6), this condition is satisfied if $\alpha>\mathcal{O}\left(10^{-17}\right)$ when $m_{A} \simeq \Delta t^{-1} \simeq H_{\text {inf }} \simeq 10^{13} \mathrm{GeV}$. Since almost all of the cosmic entropy is generated by the decay of $A$, the ratio of the energy density of $X$ to the entropy density is conserved after $A$ decay. According to Eqs. (3) and (5), the energy density of $X$ at $t=t_{d}$ is

$$
\left.\rho_{X}\right|_{d}=\mathcal{C} \alpha e^{-4 m_{X} \Delta t} m_{A} m_{X}^{2} H_{\mathrm{inf}},
$$

and the entropy density is

$$
\left.s\right|_{d}=\frac{2 \pi^{2}}{45} g_{* d} T_{d}^{3},
$$

where $g_{* d}$ and $T_{d}$ denote the effective degrees of freedom (d.o.f.), which we take as the standard value 106.75, and the temperature at the time $A$ decays, respectively. According to Eqs. (3) and (5), the latter is given by

$$
T_{d}=5 \times 10^{-2} \alpha^{1 / 4} e^{-m_{A} \Delta t} m_{A}^{3 / 4} H_{\mathrm{inf}}^{1 / 4},
$$

and, hence, the ratio of $\rho_{X}$ to $s$ is given by

$$
\frac{\rho_{X}}{s}=4 \times 10^{-2} \alpha^{1 / 4} e^{\left(3 m_{A}-4 m_{X}\right) \Delta t} \frac{m_{X}^{2} H_{\mathrm{inf}}^{1 / 4}}{m_{A}^{5 / 4}} .
$$

This should be equal to $\approx 4 \times 10^{-10} \mathrm{GeV}$ in order to explain the present dark matter density [1].

\section{CONCEALING GRAVITON}

The gravitational particle creation mechanism also produces the graviton, whose abundance is twice as much as that of a massless minimally coupled scalar particle. Since the graviton is also decoupled from the thermal bath throughout cosmic history, it increases the effective degree of relativistic freedom, and in the same way as extra species of massless neutrinos, its abundance is constrained by the big bang nucleosynthesis [30,31] and observation of the CMB [1]. The effective d.o.f. induced by the graviton at the photon decoupling are quantified as [32]

$$
N_{G W, \text { eff }}=\left.\frac{4}{7}\left(\frac{4}{11}\right)^{-4 / 3} g_{* \mathrm{DC}}\left(\frac{g_{* \mathrm{DC}}}{g_{* d}}\right)^{1 / 3}\left(\frac{\rho_{G W}}{\rho_{A}}\right)\right|_{d}
$$

in terms of extra "neutrino" generation, where $g_{* \mathrm{DC}}$ is the effective d.o.f. at the photon decoupling and $\left.\rho_{G W}\right|_{d}$ and $\left.\rho_{A}\right|_{d}$ denote the energy density of the graviton and $A$ at the time of $A$ decay, respectively. Since $g_{* \mathrm{DC}}=3.38$ and $g_{* d}=$ 106.75, Eq. (11) is rewritten as $N_{G W, \text { eff }}=\left.2.4\left(\rho_{G W} / \rho_{A}\right)\right|_{d}$. Here, $\left.\rho_{G W}\right|_{d}$ is given as [19]

$$
\left.\rho_{G W}\right|_{d} \simeq \frac{9 H_{\mathrm{inf}}^{4}}{16 \pi^{2}} a_{d}^{-4} .
$$

According to Ref. [1], $N_{G W \text {,eff }}$ must be less than 0.72, and thus Eqs. (3), (5), (11), and (12) yield a constraint:

$$
\alpha^{-1 / 3} e^{-4 m_{A} \Delta t}\left(\frac{m_{A}}{H_{\text {inf }}}\right)^{5 / 3}>2.3 \times 10^{3} .
$$

In most of the relevant inflationary models, the transition from inflation to kination takes place around the Hubble time. If $\Delta t=1.0 H_{\mathrm{inf}}^{-1}$, then the left-hand side of Eq. (13) has a maximum value $4.4 \times 10^{-2} \alpha^{-1 / 3}$ at $m_{A} \simeq 0.42 H_{\text {inf }}$. Hence, $\alpha<7.0 \times 10^{-15}$ is required in this condition. For the cases of $k$-inflation [16] and kinetically driven G-inflation [17], $\Delta t$ varies from 1.2 to $1.4 H_{\mathrm{inf}}^{-1}$ and the maximum value of $\alpha$ from $1.3 \times 10^{-15}$ to $2.7 \times 10^{-15}$. When $\alpha$ takes this maximum value $\mathcal{O}\left(10^{-15}\right), A$ decays just before the Universe becomes radiation dominated [Eq. (6)], and the reheating temperature reaches the order of $10^{7} \mathrm{GeV}$ [27]. The smallness of $\alpha$ can also be explained by Planckian interactions. For example, if a Yukawa interaction is Planck-suppressed as $\tilde{\lambda}\left(m_{A} / M_{\mathrm{Pl}}\right) A \bar{\Psi} \Psi$, the upper bound of the Yukawa coupling $\tilde{\lambda}$ becomes just an order of unity.

The parameter region allowed by Eq. (13) is shown in Fig. 1 assuming $\Delta t=1.0 H_{\text {inf }}^{-1}$. There, we have also depicted contours of $m_{X}$ which realize the proper abundance of CDM based on Eq. (10). $m_{X}$ takes the minimum value $5.8 \mathrm{TeV}$ on the edge of the allowed region. Although a lower $\alpha$ can make $m_{X}$ larger, it makes $T_{d}$ smaller much more quickly at the same time since $m_{X} \propto \alpha^{-1 / 8}$ [Eq. (10)] and $T_{d} \propto \alpha^{1 / 4}$ [Eq. (9)]. Therefore, $\alpha$ should be around the maximum value in order to sustain a sufficiently high

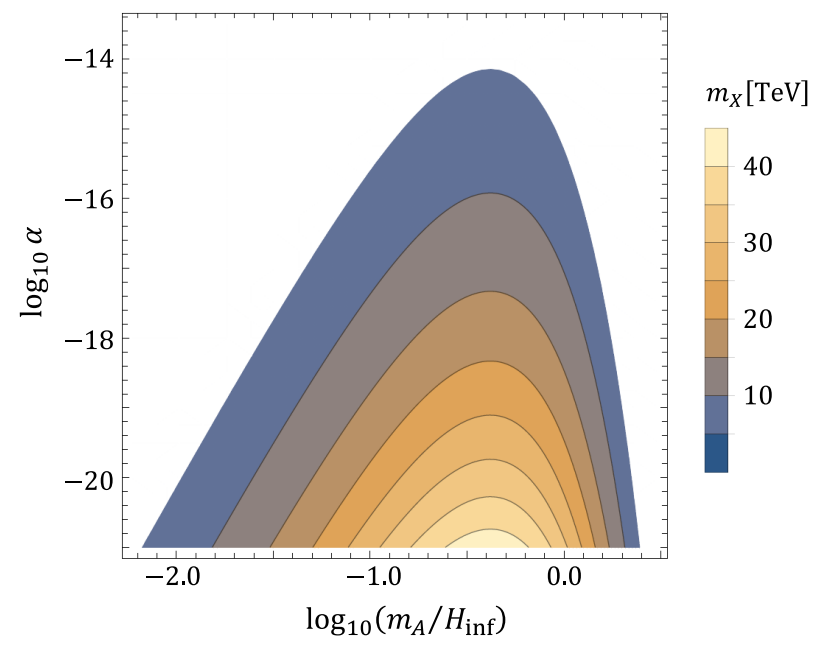

FIG. 1. Parameter values realizing the appropriate abundance of CDM while concealing the effect of gravitationally produced gravitons with $H_{\text {inf }}=10^{13} \mathrm{GeV}$ and $\Delta t=1.0 H_{\text {inf }}^{-1}$. The colored region is consistent with the $\mathrm{CMB}$ observation [1]. The maximum allowed value of $\alpha$ is $7.0 \times 10^{-15}$ with $m_{A}=0.42 H_{\text {inf }}$. The minimum value of $m_{X}$ is $5.8 \mathrm{TeV}$ on the edge of the allowed region. 
reheating temperature. We assume that $m_{X}=\mathcal{O}\left(10^{3}\right) \mathrm{GeV}$ hereafter. In terms of the Planck-suppressed Yukawa coupling $\tilde{\lambda}$, this assumption means $\tilde{\lambda} \sim \mathcal{O}(1)$.

\section{COMPARISON WITH FREEZE-IN PROCESS}

It has been claimed that PGDM may also be created by the "freeze-in" process $[6,33]$. In this section, we compare the amount of gravitationally produced particles and that of the particles produced by the freeze-in process.

Since the interaction between PGDM and other particles is so weak that PGDM never reaches thermal equilibrium, it is created at the very high-energy scale just after inflation and then no longer created or annihilated after a while. This is the freeze-in process. The effective Boltzmann equation is given in Ref. [34] as

$$
\begin{aligned}
\frac{d n_{X}}{d t} & =-3 H n_{X}-\langle\sigma v\rangle\left[n_{X}^{2}-\left(n_{X}^{\mathrm{eq}}\right)^{2}\right] \\
& \approx\langle\sigma v\rangle\left(n_{X}^{\mathrm{eq}}\right)^{2},
\end{aligned}
$$

where $n_{X}$ is the number density of $X, H$ is the Hubble parameter, $n_{X}^{\mathrm{eq}}$ is the thermal equilibrium number density of $X$, and $\langle\sigma v\rangle$ is the thermally averaged annihilation cross section. The last line comes from $n_{X} \ll n_{X}^{\mathrm{eq}} \cdot\langle\sigma v\rangle$ is given in Ref. [6] as

$\langle\sigma v\rangle=\frac{\pi m_{X}^{2}}{M_{\mathrm{Pl}}^{4}}\left[\frac{3}{5} \frac{K_{1}^{2}(z)}{K_{2}^{2}(z)}+\frac{2}{5}+\frac{4}{5} \frac{K_{1}(z)}{K_{2}(z)} z^{-1}+\frac{8}{5} z^{-2}\right]$,

where $z \equiv m_{X} / T$ is a nondimensional parameter. In our situation, $m_{X} \ll T_{d}$ and then

$$
\langle\sigma v\rangle \approx \frac{8 \pi T^{2}}{5 M_{\mathrm{Pl}}^{4}} .
$$

The temperature at time $t$ is derived from the energy density of radiation. Here, the energy density of $A$ and radiation density, $\rho_{r}=\frac{\pi^{2}}{30} g_{*} T^{4}$, obey the following Boltzmann equations:

$$
\begin{aligned}
\frac{d \rho_{A}}{d t} & =-3 H(t) \rho_{A}(t)-\Gamma \rho_{A}(t), \\
\frac{d \rho_{r}}{d t} & =-4 H(t) \rho_{r}(t)+\Gamma \rho_{A}(t) .
\end{aligned}
$$

The solution of Eqs. (17) and (18) is

$$
\rho_{r}(t)=a^{-4}(t) \int_{t_{f}}^{t} d t^{\prime} \Gamma e^{-\Gamma\left(t^{\prime}-t_{f}\right)} a\left(t^{\prime}\right) \rho_{A}\left(t_{f}\right)
$$

where $\rho_{A}\left(t_{f}\right)$ is given by Eq. (3). Since $m_{X} \ll T_{d}$ and the freeze-in process is effective only when the temperature is very high, the thermal equilibrium number density of $X$ is well approximated by $n_{X}^{\mathrm{eq}} \approx T^{3} / \pi^{2}$. Therefore, Eqs. (14), (16), and (19) give the number density of $X$ produced by the freeze-in process in one Hubble time around $t\left(<t_{d}\right)$ as

$$
\begin{aligned}
\frac{d n_{X}}{d t} H^{-1}(t) \approx & 9.4 \times 10^{-13} e^{-8 m_{A} \Delta t} \frac{\Gamma^{2} m_{A}^{4} H_{\mathrm{inf}}^{3}}{M_{\mathrm{Pl}}^{4}} \\
& \times \frac{\left(t^{4 / 3}-t_{f}^{4 / 3}\right)^{2}}{t_{f}^{2 / 3}}\left(\frac{t}{t_{f}}\right)^{-5 / 3}
\end{aligned}
$$

which is almost proportional to $t$. This means that the freeze-in process becomes more and more efficient until $t=t_{d}$. Moreover, particles produced earlier are diluted by $a^{-3}$, and, hence, it is enough to consider the freeze-in process around $t=t_{d}$. According to Eqs. (5), (14), and (16), the number density of particles produced by the freeze-in process in one Hubble time around $t=t_{d}$ is

$$
\begin{aligned}
\left.n_{X}\right|_{d} & \left.\approx\left(\frac{d n_{X}}{d t} H^{-1}\right)\right|_{d} \\
& =\frac{8 \pi T_{d}^{2}}{5 \alpha M_{\mathrm{Pl}}^{4} m_{A}}\left(n_{X}^{\mathrm{eq}}\right)_{d}^{2} \\
& =2.0 \times 10^{-12} \frac{\alpha m_{A}^{5} H_{\mathrm{inf}}^{2}}{M_{\mathrm{Pl}}^{4} e^{8 m_{A} \Delta t}} .
\end{aligned}
$$

The last line comes from Eq. (9). The produced X's have the energy around $T_{d}$. If $\alpha$ takes its maximum value $7.0 \times 10^{-15}$, then the number density of $X$ produced by the freeze-in process is $n_{X}=2.0 \times 10^{-8} \mathrm{GeV}^{3}$ at $t=t_{d}$. On the other hand, the number density of gravitationally produced particles is derived from Eq. (7) as

$$
\left.n_{X}\right|_{d} \simeq \frac{\rho_{X}}{m_{X}}=8.1 \times 10^{11} \mathrm{GeV}^{3} .
$$

Therefore, the freeze-in process is completely negligible compared with the gravitational particle creation.

\section{SUMMARY AND DISCUSSION}

We have considered two scalar fields and their gravitational creation in the inflationary model where kination follows inflation, such as in Refs. [16-18]. One of these scalar species $(A)$ decays into radiation, and the other $(X)$ is stable and remains as the PGDM. As a result, we have found the gravitational particle creation can explain sufficient reheating and the present dark matter abundance at the same time with $H_{\text {inf }} \sim 10^{13} \mathrm{GeV}, m_{A} \sim 10^{13} \mathrm{GeV}$, $\alpha \sim 10^{-15}$, and $m_{X} \sim 10^{3} \mathrm{GeV}$.

This mass range is strongly constrained for WIMPs [35]. PGDM, however, interacts with SM particles so weakly that it can escape from even the most stringent constraint. On the other hand, its feeble interaction makes it difficult to detect it experimentally. We could not help but rely on 
cosmological observations. One possibility is the size of dark matter clumps. Since PGDM has never reached kinetic equilibrium, it forms extremely small-scale clumps. The minimum size of the clump is typically determined by the comoving free streaming scale at the matter-radiation equality as [36]

$$
L_{\mathrm{fs}, \mathrm{eq}}=\int_{t_{d}}^{t_{\mathrm{eq}}} d t \frac{v(t)}{a(t)} \simeq\left(H_{\mathrm{inf}} a^{-3}\left(t_{\mathrm{RH}}\right)\right)^{-1} \ln \left(\frac{T_{\mathrm{RH}}}{T_{\mathrm{eq}}}\right)^{2},
$$

where the subscript "eq" denotes the value at the matterradiation equality. Therefore, the minimum mass of the clump is $M_{\min } \sim L_{\mathrm{fs}}^{3} \Omega_{m} \rho_{c r} \sim 2 \times 10^{-16} \mathrm{eV}$. This is much smaller even than the mass of PGDM itself, and then it means that PGDM can form any size of clumps down to a few particles. Pulsar timing array observation can detect very small-scale clumps of dark matter with masses $\sim 10^{-11}-10^{-8} M_{\odot}$ [37], and then if it detects a continuous spectrum down to a scale too small even for WIMPs or $\mathrm{PBH}$ to form, it reveals the existence of very feebly interacting dark matter-PGDM. Of course, we can also derive the constraints on Planckian interactions of dark matter from several observations, such as gamma rays, cosmic rays, neutrinos, and the CMB [38].

Finally, we comment on the effect of the nonconformal coupling. The nonconformal coupling also enhances the gravitational particle creation. In the case of the minimally coupled massive scalar field, we have found that the energy density produced by the gravitational particle creation increases only by 2 orders of magnitude when $m=$ $0.42 H_{\text {inf }}$ compared with the conformally coupled case. Hence, it does not change the situation dramatically.

\section{ACKNOWLEDGMENTS}

We acknowledge the useful comments of Toyokazu Sekiguchi and an anonymous referee. S. H. was supported by the Advanced Leading Graduate Course for Photon Science (ALPS). The work of J. Y. was supported by JSPS KAKENHI, Grant No. JP15H02082 and by a Grant on Innovative Areas No. JP15H05888.
[1] N. Aghanim et al. (Planck Collaboration), arXiv:1807.06209.

[2] D. J. H. Chung, E. W. Kolb, and A. Riotto, Phys. Rev. D 59, 023501 (1998).

[3] D. J. H. Chung, E. W. Kolb, and A. Riotto, Phys. Rev. Lett. 81, 4048 (1998).

[4] V. Kuzmin and I. Tkachev, Phys. Rev. D 59, 123006 (1999).

[5] D. J. H. Chung, P. Crotty, E. W. Kolb, and A. Riotto, Phys. Rev. D 64, 043503 (2001).

[6] M. Garny, M. Sandora, and M. S. Sloth, Phys. Rev. Lett. 116, 101302 (2016).

[7] Y. Tang and Y.-L. Wu, Phys. Lett. B 758, 402 (2016).

[8] Y. Ema, K. Nakayama, and Y. Tang, J. High Energy Phys. 09 (2018) 135.

[9] K. Sato and J. Yokoyama, Int. J. Mod. Phys. D 24, 1530025 (2015).

[10] K. Sato, Mon. Not. R. Astron. Soc. 195, 467 (1981).

[11] A. H. Guth, Phys. Rev. D 23, 347 (1981).

[12] A. D. Linde, Phys. Lett. 108B, 389 (1982);

[13] A. D. Linde, Phys. Lett. 129B, 177 (1983).

[14] L. F. Abbott, E. Farhi, and M. B. Wise, Phys. Lett. 117B, 29 (1982).

[15] A. D. Dolgov and A. D. Linde, Phys. Lett. 116B, 329 (1982).

[16] C. Armendariz-Picon, T. Damour, and V. F. Mukhanov, Phys. Lett. B 458, 209 (1999).

[17] T. Kobayashi, M. Yamaguchi, and J. Yokoyama, Phys. Rev. Lett. 105, 231302 (2010).
[18] P. J. E. Peebles and A. Vilenkin, Phys. Rev. D 59, 063505 (1999).

[19] T. Kunimitsu and J. Yokoyama, Phys. Rev. D 86, 083541 (2012).

[20] H. B. Moghaddam, R. Brandenberger, and J. Yokoyama, Phys. Rev. D 95, 063529 (2017).

[21] L. Parker, Phys. Rev. 183, 1057 (1969).

[22] Ya. B. Zeldovich and A. A. Starobinsky, Zh. Eksp. Teor. Fiz. 61, 2161 (1971) [Sov. Phys. JETP 34, 1159 (1972)].

[23] N. D. Birrell and P. C. W. Davies, J. Phys. A 13, 2109 (1980).

[24] M. S. Turner and L. M. Widrow, Phys. Rev. D 37, 3428 (1988).

[25] D. J. H. Chung, P. Crotty, E. W. Kolb, and A. Riotto, Phys. Rev. D 64, 043503 (2001).

[26] D. J. H. Chung, E. W. Kolb, and A. J. Long, J. High Energy Phys. 01 (2019) 189.

[27] S. Hashiba and J. Yokoyama, J. Cosmol. Astropart. Phys. 01 (2019) 028.

[28] V. K. Dubrovich, D. Fargion, and M. Yu. Khlopov, Astropart. Phys. 22, 183 (2004).

[29] M. Garny, A. Palessandro, M. Sandora, and M. S. Sloth, J. Cosmol. Astropart. Phys. 01 (2019) 021.

[30] A. A. Starobinsky, JETP Lett. 30, 682 (1979).

[31] H. Tashiro, T. Chiba, and M. Sasaki, Classical Quantum Gravity 21, 1761 (2004).

[32] T. Nakama and J. Yokoyama, arXiv:1803.07111. 
[33] L. J. Hall, K. Jedamzik, J. March-Russell, and S. M. West, J. High Energy Phys. 03 (2010) 080.

[34] G. F. Giudice, E. W. Kolb, and A. Riotto, Phys. Rev. D 64, 023508 (2001).

[35] E. Aprile et al. (XENON Collaboration), Phys. Rev. Lett. 121, 111302 (2018).
[36] A. Schneider, R. E. Smith, and D. Reed, Mon. Not. R. Astron. Soc. 433, 1573 (2013).

[37] K. Kashiyama and M. Oguri, arXiv:1801.07847.

[38] Y. Mambrini, S. Profumo, and F. S. Queiroz, Phys. Lett. B 760, 807 (2016). 